\title{
New upper bounds on separating codes
}

\author{
Gérard Cohen ${ }^{1}$ \\ Ecole Nationale Supérieure \\ des Télécommunications \\ 46 rue Barrault \\ 75634 Paris, France \\ e-mail: cohen@inf.enst.fr
}

\author{
Hans Georg Schaathun \\ Department of Informatics \\ University of Bergen \\ Høyteknologisenteret \\ N-5020 Bergen, Norway \\ e-mail: georg@ii.uib.no
}

\begin{abstract}
Separating codes, initially introduced to test automaton, have revived lately in the study of fingerprinting codes, which are used for copyright protection. Separating codes play their role in making the fingerprinting scheme secure agains coalitions of pirates. We provide here better bounds on such codes.

Index Terms- separating code, copyright protection.
\end{abstract}

\section{INTRODUCTION}

Separating codes were introduced in 1969 and have been the topic of several papers with various motivations. Many initial results are due to Sagalovich; see [5] for a survey, and also $[4,8]$. New applications of separating codes have appeared during the last decade, namely traitor tracing and fingerprinting.

Fingerprinting is a proposed technique for copyright protection. The vendor has some copyrighted work of which he wants to sell copies to customers. If he is not able to prevent the customer from duplicating his copy, he may individually mark every copy sold with a unique fingerprint. If an illegal copy (for which the vendor has not been paid) subsequently appears, it may be traced back to one legal copy and one pirate via the fingerprint. A pirate is here any customer guilty of illegal copying of the copyrighted work.

Traitor tracing is the same idea applied to broadcast encryption keys. E.g. the vendor broadcasts encrypted pay-TV, and each customer buys or leases a decoder box to be able to decrypt the programmes. If the vendor is not able to make the decoder completely tamperproof, he may fingerprint the decryption keys which are stored in the box.

\footnotetext{
${ }^{1}$ Corresponding author
}

The set of fingerprints in use, is called the fingerprinting code. Separating codes are used in the study of collusion secure fingerprinting codes. If several pirates collude, they posess several copies with different fingerprints. By comparing their copies, they will find differences which must be part of the fingerprint. These identified 'marks' may be changed to produce a false fingerprint. A collusion secure code should aim to identify at least one of the pirates from this false fingerprint.

We shall introduce two useful concepts regarding collusion secure code. If the code is $t$-frameproof, it is impossible for any collusion of at most $t$ pirates to produce a false fingerprint which is also a valid fingerprint of an innocent user. In other words, no user may be framed by a coalition of $t$ pirates or less. A $t$-frameproof code is the same as a $(t, 1)$ separating code, which will be defined formally in the next section.

If the code is $t$-identifying, the vendor is always able to identify at least one pirate from any coalition of size at most $t$, given a false fingerprint created by the coalition. Good $t$-identifying codes are rare, so we are also interested in probabilistic $(t, \epsilon)$ identifying codes, where the vendor is able to identify a pirate with probability $1-\epsilon$ for some small $\epsilon$. Barg et al. [1] apply $(t, t)$-separating codes to construct $(t, \epsilon)$-identifying codes.

\section{DEFINITIONS}

For any positive real number $x$ we denote by $\lceil x\rceil$ the smallest integer at least equal to $x$. Let $G F(q)$ be a finite field of $q$ elements, and $G F(q)^{n}$ the $n$ dimensional vector space thereover. A subset $C \subseteq$ $G F(q)^{n}$ is called an $(n, M)$-code if $|C|=M$. For any vector $\mathbf{x} \in G F(q)^{n}$, we write $x_{i}$ for the $i$-th component, so that $\mathrm{x}=\left(x_{1}, x_{2}, \ldots, x_{n}\right)$.

Consider a subset $\mathcal{C} \subseteq C$. For any position $i$, we define the projection $P_{i}(\mathcal{C})=\bigcup_{\mathrm{a} \in \mathcal{C}}\left\{a_{i}\right\}$. The feasible 
set of $\mathcal{C}$ is

$$
F(\mathcal{C})=\left\{\mathrm{x} \in G F(q)^{n}: \forall i, x_{i} \in P_{i}(\mathcal{C})\right\} .
$$

If $\mathcal{C}$ is the fingerprints held by some pirate coalition, then $F(\mathcal{C})$ is the set of fingerprints they may produce. If two non-intersecting coalitions can produce the same descendant, i.e., if their feasible sets intersect, it will be impossible to trace with certainty even one pirate. This motivates the following defnition.

Definition $1 A$ code $C$ is $\left(t, t^{\prime}\right)$-separating if, for any pair $\left(T, T^{\prime}\right)$ of disjoint subsets of $C$ where $|T|=$ $t$ and $\left|T^{\prime}\right|=t^{\prime}$, the feasible sets are disjoint, i.e. $F(T) \cap F\left(T^{\prime}\right)=\emptyset$.

Since the separation property is preserved by translation, we shall always assume that $0 \in C$. The separation property can be rephrased as follows when $q=2$ : For any ordered $t+t^{\prime}$-tuple of codewords, there is a coordinate where the $t+t^{\prime}$-tuple $(1 . .10 .0)$ of weight $t$ or its complement occurs.

\section{UPPER BOUNDS ON $(t, 1)$ SEPARATING CODES}

The case $t^{\prime}=1$ corresponds to "frameproof" codes introduced in [2]. Körner (personal communication) has a simplified proof of $R \leq 1 / 2$ for $(1,2)$-separation in the binary case. We generalize it to any $t$ and $q$ as follows.

Partition $\{1,2, . . n\}$ into $t$ almost equal parts $P_{1}, \ldots, P_{t}$ of size approximately $n / t$. Say a codeword $\mathrm{c}$ is unmatched on $P_{i}$ if no other codeword projects onto the same $n / t$-tuple on $P_{i}$. Denote by $U_{i}$ the subset of codewords unmatched on $P_{i}$.

Lemma 1 If $C$ is $(1, t)$-separating, then every codeword $c$ of $C$ is unmatched on at least one $P_{i}$.

Proof: Otherwise, $c$ could not be separated form the at most $t$ codewords matching it on the $P_{i}$ 's.

Combining $C=\bigcup U_{i}$ with $\left|U_{i}\right| \leq q^{[n / t]}$, we get

Theorem 1 If $C$ is $(1, t)$-separating, then $|C| \leq$ $t q^{\lceil n / t\rceil}$.

For constant $t$, this asymptotically gives $R \leq 1 / t$. This is essentially tight, as we now show.

To get infinite constructions of separating codes, we need the following constructive result from Tsfasmann [10].
Lemma 2 For any $\alpha>0$ there is an infinite family of $q$-ary codes with parameters $\left(N, q^{N R}, N \delta\right)$ for $N \geq$ $N_{0}(\alpha)$ and

$$
R+\delta \geq 1-(\sqrt{q}-1)^{-1}-\alpha
$$

Together with the following easily checked sufficient condition in terms of minimum distance for a code to be $t$-separating:

$$
t d>(t-1) n
$$

this yields

Theorem 2 For fixed $t$ and large enough $q$, the largest possible rate of a q-ary family of $t$-separating codes satisfy

$$
R=(1 / t)(1+o(1))
$$

\section{THE GENERAL CASE}

\section{A Separating Weights}

Given a $\left(t, t^{\prime}\right)$-configuration $\left(T, T^{\prime}\right)$ we define the separating set $\Theta\left(T, T^{\prime}\right)$ to be the set of coordinate positions where $\left(T, T^{\prime}\right)$ is separated. Let $\theta\left(T, T^{\prime}\right):=\# \Theta\left(T, T^{\prime}\right)$ be the separating weight. Clearly $\theta\left(T, T^{\prime}\right) \geq 1$ is equivalent with $\left(T, T^{\prime}\right)$ being separated. The minimum $\left(t, t^{\prime}\right)$-separating weight $\theta_{t, t^{t}}(C)$ is the least separating weight of any $\left(t, t^{\prime}\right)$-configuration of $C$. The minimum separating weights have previously been studied by Sagalovich [5]. Clearly $\theta_{1,1}(C)=d_{1}(C)$.

\section{$B$ A stronger property in the binary case}

Definition 2 (Completely Separating Code) $A$ binary code is said to be $\left(t, t^{\prime}\right)$-completely separating $\left(\left(t, t^{\prime}\right)-C S S\right)$ if for any set ordered set of $t+t^{\prime}$ codewords, there is at least one column with 1 in the $t$ upper positions, and 0 elsewhere, and one column with 0 in the $t$ upper positions and 0 in the $t^{\prime}$ lower ones.

We define $R_{S S}\left(t, t^{\prime}\right)$ as the largest asymptotical possible rate of a family of $\left(t, t^{\prime}\right)$-SS, and similarly define $R_{C S S}\left(t, t^{\prime}\right)$ for $\left(t, t^{\prime}\right)$-CSS. We clearly obtain that

$$
R_{S S}\left(t, t^{\prime}\right) \geq R_{C S S}\left(t, t^{\prime}\right) \geq \frac{1}{2} R_{S S}\left(t, t^{\prime}\right)
$$


$C \quad$ Improved upper bounds on $(t, t)$-separating codes

Theorem 3 Any $(t, t)$-separating $\left(\theta_{0}, M, \theta_{1}\right)$ code with separating weights $\left(\theta_{1}, \ldots, \theta_{t}\right)$ gives rise to a completely $(i, i)$-separating $\left(\theta_{t-i}, M-2 t+2 i, 2 \theta_{t+1-i}\right)$ code with complete-separating weight $\theta_{t}$, for any $i<$ $t$.

Proof: Consider a pair of $(t-i)$-tuples of vectors which are separated on $\theta_{t-i}$ positions. Pick any vector $\mathbf{c}$ from the first $(t-i)$-tuple and replace the code $C$ by its translation $C-\mathbf{c}$. Thus all the columns which separates the two tuples have the form $(0 \ldots 01 \ldots 1)$.

Now consider any two $i$-tuples of vectors. Coupling each $i$-tuple with a $(t-1)$-tuple, we get two $t$-tuples which must be separated on $\theta_{t}$ positions, i.e. the two $i$-tuples must have at least $\theta_{t}$ columns of the form $(0 \ldots 01 \ldots 1)$. Now, observe that we can swap the two $(t-i)$-tuples, and the two resulting $t$-tuples are still separated. This guarantees at least $\theta_{t}$ columns of the form $(1 \ldots 10 \ldots 0)$.

Deleting all the columns where the two $(t-i)$ tuples are not separated, and the words of these two tuples must this leave us with an $(i, i)$-CSS with separating weight $\theta_{i}$ and parameters $\left(\theta_{t-i}, M-2 t+\right.$ $\left.2 i, 2 \theta_{t+1-i}\right)$, as required.

Theorem 4 Any completely $(t, t)$-separating $\left(\theta_{0}, M, 2 \theta_{1}\right)$ code with separating weights $\left(\theta_{1}, \ldots, \theta_{t}\right)$ gives rise to a completely $(i, i)$-separating $\left(\theta_{t-i} ; M-2 t+2 i, 2 \theta_{t+1-i}\right)$ code with completeseparating weight $\theta_{i}$, for any $i<t$.

This is proved in the same way as the previous theorem.

Theorem 5 For any $(t, t)$-CSS, the rate $R_{t}$ satisfies

$$
R_{t} \leq \vec{R}\left(2 R_{t} / \bar{R}_{t-1}\right)
$$

where $\bar{R}(\delta)$ is any upper bound on the rate of errorcorrecting codes in terms of the normalised minimum distance, and $\tilde{R}_{t-1}$ is the upper bound on the rate of any $(t-1, t-1)-C S S$.

Proof: Let $C_{t-1}$ be the $(t-1, t-1)$-CSS which exists by Theorem 4 , and let $R_{t-1}$ be its rate. We have that

$$
\delta_{t}=2 \frac{\theta_{1}}{\theta_{0}}=2 \frac{\log M}{\theta_{0}} \frac{\theta_{1}}{\log M}=2 R_{t} / R_{t-1} .
$$

Now, obviously $R_{t} \leq \bar{R}\left(\delta_{t}\right)$, which is decreasing in $\delta_{t}$, and this gives the result.

With a completely analogous proof, we also get the following.

Theorem 6 For any $(t, t)-S S$, the rate $R$ satisfies

$$
R \leq \bar{R}\left(R / \bar{R}_{t-1}\right),
$$

where $\vec{R}(\delta)$ is any upper bound on the rate of errorcorrecting codes in terms of the normalised minimum distance, and $\bar{R}_{t-1}$ is the upper bound on the rate of any $(t-1, t-1)-C S S$.

Putting equality in the bounds and solving the equations, we get the upper rate bounds given as 'Bound 1' in Table 1. Comparing this to the CSS bounds by D'yachkov et al., we see that we get an improvement from $(3,3)$-CSS and onwards. However, D'yachkov et al. [9] have a good bound on $(2,2)$-CSS, and using that as a seed for the recursive bounds of our theorems, we get the better bounds given as 'Bound 2' in the table.

Example 1 Let $C_{1}$ be an asymptotic class of $\left(\theta_{0}, 2^{k}, \theta_{1}\right)(3,3)-S S$. Then there is an asymptotic class $C_{2}$ of $\left(\theta_{1}, 2^{k}, \theta_{2}\right)(2,2)-C S S$. We have that $R_{2}=k / \theta_{1} \leq 0.161$, and

$$
R_{1}=k / \theta_{0}=R_{2} \delta_{1} \leq 0.161 \delta_{1}
$$

which is equivalent to

$$
\delta_{1} \geq R_{1} / 0.161
$$

We can use any upper bound $\vec{R}(\delta)$ on $R_{1}$, and get

$$
R_{1} \leq \bar{R}\left(\delta_{1}\right) \leq \bar{R}\left(R_{1} / 0.161\right) .
$$

Using the linear programming bound, we get $R_{1} \leq$ 0.0663 .

\section{REFERENCES}

[1] A. Barg, G. Blakley and G. Kabatiansky, "Digital fingerprinting codes", DIMACS Technical Report 200152 (2001).

[2] D. Boneh and J. Shaw, "Collusion-secure fingerprinting for digital data", IEEE Trans. on Inf. Theory, 44 (1998), pp. 480-491.

[3] B. Chor, A. Fiat and M. Naor, "Tracing traitors", Crypto'94 LNCS 839 (1994), pp. 257-270. 


\begin{tabular}{|c||l|l||l||l|l|}
\hline \multicolumn{1}{|c||}{} & \multicolumn{2}{c||}{ Bound 1 } & \multicolumn{2}{c||}{$\begin{array}{l}\text { D'yachkov et al. } \\
\text { CSS rate }\end{array}$} & \multicolumn{2}{c|}{ Bound 2 } \\
$(t, t)$ & CSS rate & SS rate \\
\hline \hline$(1,1)$ & 1 & 1 & 1 & 1 & 1 \\
$(2,2)$ & 0.1712 & 0.2835 & 0.161 & - & - \\
$(3,3)$ & 0.03742 & 0.06998 & 0.0445 & 0.0354 & 0.0663 \\
$(4,4)$ & 0.008843 & 0.01721 & 0.0123 & 0.00837 & 0.0163 \\
$(5,5)$ & 0.002156 & 0.004261 & 0.00333 & 0.00204 & 0.00404 \\
\hline
\end{tabular}

Table 1: Rate bounds on CSS and SS.

[4] J. Körner and G. Simonyi, "Separating partition systems and locally different sequences," SIAM J. Discrete Math., 1 No 3 (1988) pp. 355-359.

[5] Yu. L. Sagalovich, "Separating systems", Probl. Inform. Trans. 30 No 2 (1994) pp. 105-123.

[6] D.R. Stinson and R. Wei, "Combinatorial properties and constructions of traceability schemes and frameproof codes", SIAM J. Discrete Math., 11 (1998) pp. 41-53.

[7] A. Barg, G. Cohen, S. Encheva, G. Kabatiansky and G. Zémor, "A hypergraph approach to the identifying parent property", SIAM J. Disc. Math., vol. 14, 3 (2001) pp.423-431.

[8] G. Cohen, S. Encheva and H.-G. Schaathun, "More on $(2,2)$-separating systems", IEEE Trans. Inform. Theory, vol. 48, 9 (2002) pp. 2606-2609.

[9] A. D'yachkov, P. Vilenkin, A. Macula and D. Torney, "Families of finite sets in which no intersection of $\ell$ sets is covered by the union of $s$ others", J. Combinatorial Theory, vol. 99, 195-208 (2002).

[10] M.A. Tsfasmann, "Algebraic-geometric codes and asymptotic problems", Discrete Applied Math., vol. 33 (1991) pp. 241-256. 